\title{
Cytokine gene expression signature in ovarian clear cell carcinoma
}

\author{
NOZOMU YANAIHARA ${ }^{1}$, MICHAEL S. ANGLESIO ${ }^{2}$, KAZUNORI OCHIAI $^{1}$, YUKIHIRO HIRATA $^{1}$, \\ MISATO SAITO $^{1}$, CHIE NAGATA $^{1}$, YASUSHI IIDA ${ }^{1}$, SATOSHI TAKAKURA ${ }^{1}$, \\ KYOSUKE YAMADA $^{1}$, TADAO TANAKA ${ }^{1}$ and AIKOU OKAMOTO ${ }^{1}$ \\ ${ }^{1}$ Department of Obstetrics and Gynecology, The Jikei University School of Medicine, Tokyo, Japan; \\ ${ }^{2}$ Department of Pathology, University of British Columbia, Vancouver, British Columbia, Canada
}

Received March 16, 2012; Accepted May 18, 2012

DOI: $10.3892 /$ ijo.2012.1533

\begin{abstract}
Cytokine expression in a tumor microenvironment can impact both host defense against the tumor and tumor cell survival. In this study, we sought to clarify whether the cytokine gene expression profile could have clinical associations with ovarian cancer. We analyzed the expression of 16 cytokine genes $(I L-1 \alpha, I L-1 \beta, I L-2, I L-4, I L-5, I L-8, I L-10, I L-12 p 35$, IL-12p40, IL-15, IFN- $\gamma, T N F-\alpha, I L-6, H L A-D R A, H L A-D P A I$ and $C S F 1$ ) in 50 ovarian carcinomas. Hierarchical clustering analysis of these tumors was carried out using Cluster software and differentially expressed genes were examined between clear cell carcinoma (CCC) and other subtypes. Following this examination we evaluated the biological significance of $I L-6$ knockdown in CCC. Unsupervised hierarchical clustering analysis of cytokine gene expression revealed two distinct clusters. The relationship between the two clusters and clinical parameters showed statistically significant differences in CCC compared to other histologies. CCC showed a dominant Th-2 cytokine expression pattern driven largely by $I L-6$ expression. Inhibition of IL-6 in CCC cells suppressed Stat 3 signaling and rendered cells sensitive to cytotoxic agents. The unique cytokine expression pattern found in CCC may be involved in the pathogenesis of this subtype. In particular, high $I L-6$ expression appears likely to be driven by the tumor cells, fueling an autocrine pathway involving IL-6 expression and Stat 3 activation and may influence survival when exposed to cytotoxic chemotherapy. Modulation of IL-6 expression or its related signaling pathway may be a promising strategy of treatment for CCC.
\end{abstract}

\section{Introduction}

Ovarian cancer is the most lethal gynecological malignancy worldwide; in Japan alone it is estimated that there were 7913 new cases of ovarian cancer and 4415 cases of mortality

Correspondence to: Dr Nozomu Yanaihara, Department of Obstetrics and Gynecology, The Jikei University School of Medicine 3-25-8 Nishi-Shinbashi, Minato-ku, Tokyo 105-8461, Japan

E-mail: yanazou@jikei.ac.jp

Key words: cytokine, expression signature, IL-6, clear cell carcinoma, stat 3 due to this disease in 2006 (Center for Cancer Control and Information Services, National Cancer Center, Japan). Overall, ovarian cancer patients respond to cytoreductive surgery and platinum and taxane based combination chemotherapy, however, advanced cases have a high recurrence rate and the 5 -year survival rate has remained largely unchanged since the 1980s, being close to 30\% (1). This, however, is skewed, given the mass of recent evidence suggesting that histological subtypes of ovarian cancer represent unique diseases, that the majority of cases (50-70\%) are of serous histology, and that all ovarian carcinoma subtypes are still treated with a 'one size fits all' approach. Serous cancers seem to initially respond well to platinum and taxane chemotherapy, whereas other subtypes respond poorly or not at all (2-5). These data are finally initiating separate clinical trials for unique subtypes such as clear cell carcinoma (CCC) (6) and mucinous adenocarcinoma (MA) (7). Even within histologically defined groups, such as high-grade serous tumors, molecular subtypes are emerging. Defining molecular mechanisms involved in the development and progression of ovarian cancer and their interaction with host defenses is integral to the development of novel therapeutic approaches and successful treatment stratification.

Factors intrinsic to the tumor and the host influence progression, host defense against tumors is controlled by several immunological mediators that play an important role in the host-tumor immune system conflict (8). The hosttumor immune response is in part regulated by CD4 helper T-lymphocytes, Th1 and Th2 cells. Th1 cells produce proinflammatory cytokines and drive the cell-mediated immune response, while Th 2 cells regulate humoral immunity by expressing anti-inflammatory cytokines. Alterations of cytokine expression and an imbalance in Th1/Th2 cytokine response have previously been observed in ovarian carcinomas (9-11). Serous adenocarcinoma (SA) is frequently subject to infiltration by activated T cells (TILs; tumor infiltrating lymphocytes), and patients with dense infiltrates of $\mathrm{CD}^{+}$ $\mathrm{CD}^{+} \mathrm{T}$ cells had a more favorable prognosis while infiltration of other cell types, including $\mathrm{CD} 4{ }^{+} \mathrm{CD} 25^{+} \mathrm{FoxP}^{+}$regulatory T cells, opposed antitumor immunity (9).

Seike et al reported that a unique cytokine gene expression signature of noncancerous lung tissue and corresponding tumor tissue in lung adenocarcinoma predicted metastasis and disease progression (12). The prognostic signature consisted mainly of cytokine genes that were expressed in Th1 and Th2 
cells. Taken together, these findings suggest that alterations in cytokine gene expression of tumors could be involved in the pathogenesis of other types of cancer, including ovarian cancer.

In this study, we sought to clarify whether the cytokine gene expression profile could have clinical associations with ovarian cancer by focusing on the expression profile of 12 cytokine genes in 50 ovarian cancers. We found that a cytokine gene expression signature of ovarian cancer could distinguish the histological subtype, and a unique expression pattern found in CCC may be involved in the pathogenesis of this ovarian cancer subtype. We further evaluated the biological significance of $I L-6$ overexpression in CCC using siRNA and found that the drug resistance phenotype in $\mathrm{CCC}$ might be regulated by the activated IL- 6 signaling pathway including Stat 3 activation.

\section{Materials and methods}

Clinical samples and cell lines. Tumor specimens were surgically obtained from patients with primary ovarian carcinoma who were treated at the Department of Obstetrics and Gynecology, The Jikei University School of Medicine. The Jikei University School of Medicine Ethics Review Committee approved the study protocol and informed consent was obtained from all patients. Tumors were staged in accordance with the International Federation of Gynecology and Obstetrics (FIGO) system (1988). Fifty tumor RNA specimens that passed quality control standards were used to identify a gene signature. Clinical and pathological characteristics of the 50 patients are shown in Table I. Forty-seven patients received first-line platinum-based chemotherapy.

Seven human CCC cell lines (JHOC-5, JHOC-7, JHOC-8, JHOC-9, HAC-2, RMG-I, and RMG-II) and five human ovarian non-CCC cell lines were used in this study. JHOC-5, JHOC-7, JHOC-8, and JHOC-9 were obtained from Riken BioResource center (Tsukuba, Japan). HAC-2 was kindly provided by Dr M. Nishida (Tsukuba University, Tsukuba, Japan). RMG-I and RMG-II were kindly provided by Dr D. Aoki (Keio University, Tokyo, Japan). A2780 (undifferentiated carcinoma) was provided by Dr E. Reed (NCI, Bethesda, MD) and 2008 (SA) was provided by Dr S.B. Howell (UCSD, San Diego, CA). SKOV3 (SA), MCAS (MA), and Tyk-nu (undifferentiated carcinoma) were obtained from ATCC (Rockville, MD). RMG-I and RMG-II were maintained in Ham's F12 medium (Sigma-Aldrich, Tokyo, Japan) with $10 \%$ fetal bovine serum (FBS), and the other cells were cultured in RPMI-1640 (Sigma-Aldrich) containing 10\% FBS.

Quantitative reverse transcription-polymerase chain reaction analysis. All tissue was freshly collected during surgery and stored at $-80^{\circ} \mathrm{C}$. Cryostat sections containing $>80 \%$ cancer cells were prepared as tumor specimens. Total RNA was isolated with TRIzol reagent (Invitrogen, Carlsbad, CA). Total RNA (3 $\mu \mathrm{g}$ ) was converted to complementary DNA (cDNA) with random hexamers and SuperScript III FirstStrand Synthesis kit (Invitrogen). The cDNAs were then used for qRT-PCR analysis of 16-gene expression profile. Normal ovarian tissue from a patient who had undergone surgical resection was used as a reference for each tissue sample.
Table I. Clinical and pathological characteristics of the 50 ovarian cancer patients.

\begin{tabular}{lrc}
\hline Parameters & No. of patients & $\%$ of patients \\
\hline Patient age & & \\
$\leq 60$ years & 41 & 82 \\
$>60$ years & 9 & 18 \\
FIGO stage & & \\
I & 19 & 38 \\
II & 3 & 6 \\
III & 22 & 44 \\
IV & 6 & 12 \\
Histological type & & \\
SA & 24 & 48 \\
EA & 5 & 10 \\
CCC & 21 & 42 \\
Residual tumor & & 64 \\
$\leq 1$ cm & 32 & 36 \\
$>1$ cm & 18 & \\
\hline
\end{tabular}

SA, serous adenocarcinoma; EA, endometrioid adenocarcinoma; $\mathrm{CCC}$, clear cell carcinoma.

Several studies have suggested that normal ovarian tissue and even surface ovarian epithelium may not be the tissue of origin for many ovarian epithelial malignancies (13), therefore the use of ovarian tissue as a reference for qRT-PCR is arbitrary and not meant for a direct comparison of subtypes to normal ovarian tissue but rather for an intercomparison of tumor samples. The expression profiles of 12 cytokine genes [i.e., interleukin $1 \alpha(I L-1 \alpha)$, interleukin $1 \beta(I L-1 \beta)$, interleukin 2 $(I L-2)$, interleukin $4(I L-4)$, interleukin $5(I L-5)$, interleukin 8 $(I L-8)$, interleukin $10(I L-10)$, interleukin 12 p35 (IL-12p35), interleukin 12 p40 (IL-12p40), interleukin 15 (IL-15), interferon $\gamma(I F N-\gamma)$ and tumor necrosis factor- $\alpha(T N F-\alpha)]$ were quantified with the use of TaqMan Cytokine Gene Expression Plates (Applied Biosystems, Foster City, CA). Additional individual Taqman assays (Applied Biosystems) were also used to measure expression of interleukin $6(I L-6)$, major histocompatibility complex (MHC) class II antigen DR $\alpha$ (HLA-DRA), MHC class II antigen DP $\alpha 1$ (HLA-DPA1), and colony stimulating factor $1(C S F 1)$. All PCR reactions were performed with a StepOnePlus Real-Time PCR System (Applied Biosystems), Human 18S ribosomal RNA (rRNA) labeled with VIC reporter dye (Applied Biosystems) was used as an endogenous control. Gene expression was quantified using the comparative method $\left(2^{-\Delta \Delta C T}\right)$, where $C_{T}=$ threshold cycle, $\Delta \Delta \mathrm{C}_{\mathrm{T}}=\left(\mathrm{C}_{\mathrm{T}}\right.$ cytokine $\left.-\mathrm{C}_{\mathrm{T}} 18 \mathrm{~S} \mathrm{rRNA}\right)-\left(\mathrm{C}_{\mathrm{T}}\right.$ reference $-\mathrm{C}_{\mathrm{T}}$ $18 \mathrm{~S}$ rRNA), as previously described (14). To ensure RNA of sufficient quality and eliminate spurious amplification artifacts, all samples were required to have average $\mathrm{CT}$ values for cytokine genes $>35$ cycles, as previously described (12).

Small interfering RNA and cytotoxicity assays. Small interfering RNA (siRNA) transfection was performed using Lipofectamine $^{\mathrm{TM}}$ RNAiMAX (Invitrogen). Briefly, HAC-2 and 


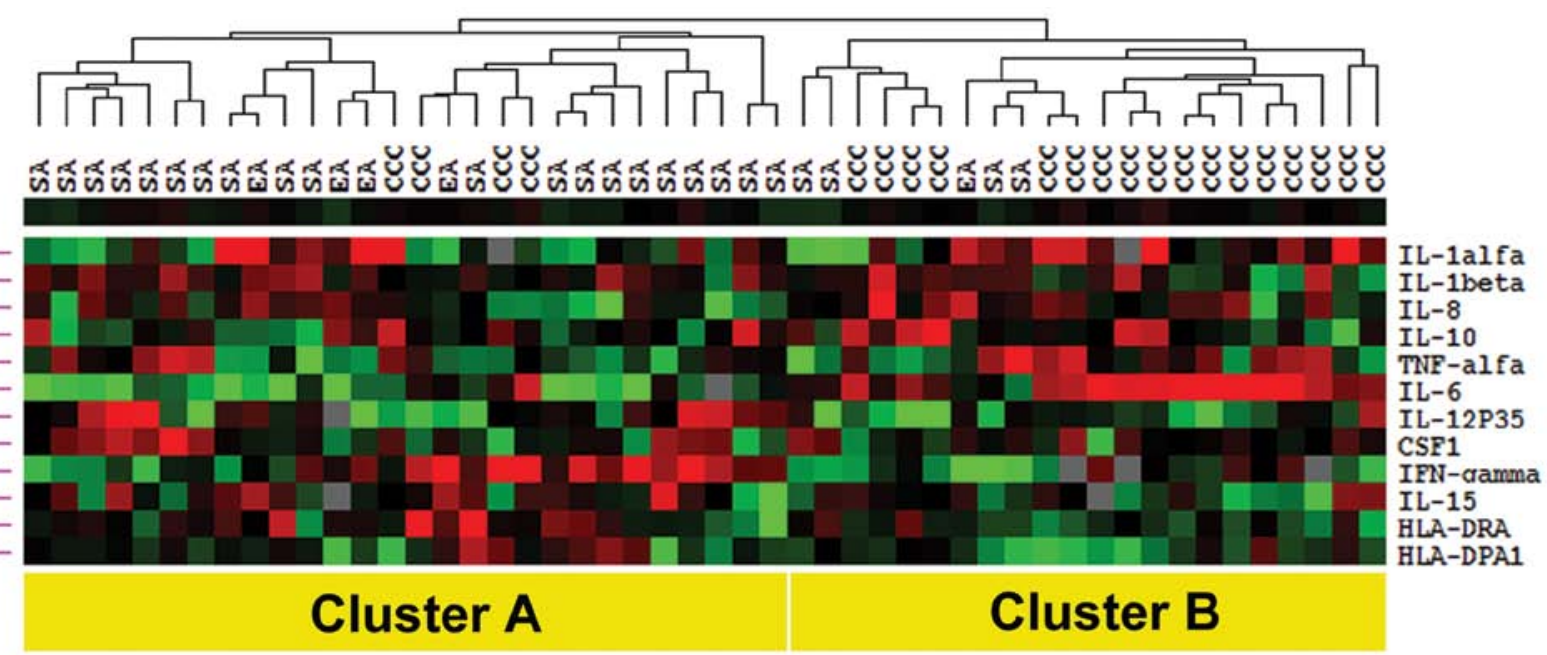

Figure 1. Expression profile of 12 cytokine genes in 50 ovarian cancer samples. Tissue samples and genes were analyzed for the calculated centered correlation distance and average linkage according to the ratios of their abundance to the median abundance of all genes among all samples. Unsupervised hierarchical clustering from these cases showed the two main trees with their corresponding histological types, i.e. cluster A and cluster B.

JHOC-5 cells were seeded at $1.5 \times 10^{5}$ in a $6 \mathrm{~cm}$ dish, validated Stealth RNAi ${ }^{\mathrm{TM}}$ siRNA for IL-6 (Invitrogen), or stealth RNAi siRNA negative control (Invitrogen), were transfected at a final concentration of $2.5 \mathrm{nM}$. After $24 \mathrm{~h}$ of transfection, cells were re-seeded in 96-well plates with various concentrations of cisplatin or paclitaxel. In vitro cytotoxicity was measured after $96 \mathrm{~h}$ by means of the MTS assay using CellTiter 96 AQueous One Solution (Promega, Madison, WI). MTS solution was added to each well and incubated for $4 \mathrm{~h}$. Absorbance was measured at $490 \mathrm{~nm}$ using a microplate reader. Data were collected as the average absorbance of three wells in any one experiment and is presented from three independent experiments; mean \pm 1 standard deviation.

Western blot analysis. Total cell lysates were prepared in $1 \mathrm{X}$ RIPA lysis buffer and protein concentration was determined by the DC Protein Assay (Bio-Rad, Hercules, CA). Total protein $(40 \mu \mathrm{g})$ was resolved on gradient NuPage $4-12 \%$ Bis-Tris gels (Invitrogen) and immunoblotted with specific antibodies: anti-Stat3 (clone 79D7; 1:2000), pStat3 (clone D3A7; 1:500), and $\beta$-actin (clone 13E5; 1:1000) from Cell Signaling Technology (Beverly, MA); anti-IL-6R $\alpha$ (clone C-20; 1:1000) from Santa Cruz Biotechnology (Santa Cruz, CA). All blots were incubated with primary antibodies diluted in TBS with $0.1 \%$ Tween-20 and 5\% bovine serum albumin overnight at $4{ }^{\circ} \mathrm{C}$ with gentle agitation. Horseradish peroxidase (HRP)-conjugated secondary antibody (Cell Signaling Technology; 1:10000) was diluted in TBS with $0.1 \%$ Tween-20 and 5\% nonfat milk for $1 \mathrm{~h}$ at room temperature with gentle agitation. Positive immunoreactions were detected using ImmunoStar LD chemiluminescence system (Wako, Tokyo, Japan).

Statistical analysis. Unsupervised hierarchical clustering analysis was performed with Gene Cluster 3.0 (http://genexpress. stanford.edu/tutorials/cluster_view.html; Stanford University, Palo Alto, CA) and visualized with Tree View (http:// genexpress.stanford.edu/tutorials/tree_view.html; Stanford University). Tissue samples and genes were analyzed for the calculated centered correlation distance and average linkage according to the ratios of their abundance to the median abundance of all genes among all samples as previously described (12). The correlation between sample clusters and clinical parameters was analyzed using the Fisher's exact test, with $\mathrm{p}<0.05$ considered to indicate statistically significant differences.

In class comparison analysis using BRB-ArrayTools, we identified genes that were differently expressed among groups using t-test with univariate permutation tests to evaluate the significance of individual genes and correct for multiple hypothesis testing. The proportion of the permutations of the class label giving a t-test $p$-value as small as obtained with the true class labels is the univariate permutation $p$-value for that gene. The false discovery rate (FDR) associated with a row of the table is an estimate of the proportion of the genes with univariate $p$-values less than or equal to the one in that row that represent false positives.

A 2-sided Student's t-test was used to evaluate the sensitivity of cytotoxic agents in ovarian cancer cell lines with $\mathrm{p}<0.05$ considered to indicate statistically significant differences.

\section{Results}

Hierarchical clustering analysis of ovarian cancer. To investigate the role of cytokine gene expression in ovarian cancer, we first analyzed the expression of 16 cytokine genes in tumor specimens from 50 primary ovarian cancers. These 16 genes were previously shown to be part of a unique inflammation/ immune response-related signature in lung adenocarcinoma patients (12). Expression of $I L-2, I L-4, I L-5$, and $I L-12 p 40$ genes was below the detectable threshold across our sample set and were therefore not carried forward in subsequent analysis. Unsupervised hierarchical clustering analysis of the 50 ovarian cancer samples, which was based on the similarities in expression patterns of the remaining 12-gene panel, revealed two distinct main clusters, namely cluster $A(n=28)$ and cluster $\mathrm{B}(\mathrm{n}=22)$ (Fig. 1). Examination of the relationship between the two clusters and clinicopathological features revealed statisti- 
cally significant differences between cluster A and cluster B with respect to histological type (Table II). Cluster A consisted of 20 SA cases (83\%), 4 EA cases $(80 \%)$ and 4 CCC cases (19\%), and the cluster B included 4 cases of SA (17\%), one EA case $(20 \%)$ and 17 cases of CCC (81\%). In addition, there was a marginally significant difference between the two clusters and FIGO stage. However, there was no statistically significant difference in age, residual tumor size, and patient prognosis between the clusters (data not shown). Of particular note, cases in cluster B showed higher expression of Th2-related cytokine genes including $I L-6, I L-8$, and $I L-10$.

Given a trend for $\mathrm{CCC}$ and non-CCC across the two clusters we further examined the normalized expression data comparing histological classes of ovarian carcinoma, CCC vs. non-CCC. Four of the twelve genes had a statistically significant difference in expression between groups at $\mathrm{p}<0.05$ with univariate permutation test (Table III). Among them, the expression level of $I L-6$ was the most significant gene in CCC compared to non-CCC.

IL-6 expression and IL-6 siRNA transfection in ovarian cancer cell lines. Next, we analyzed the $I L-6$ gene expression in twelve ovarian cancer cell lines including seven CCC cell lines. Consistent with the data obtained from surgical samples, the $I L-6$ gene expression of CCC cell lines was typically higher than that of non-CCC cell lines (Fig. 2A). To begin to address whether the endogenous production of IL- 6 by CCC could have biological significance of CCC, we inhibited expression of $I L-6$ in CCC cells using the siRNA approach and examined the effects of $I L-6$ expression on the cell growth and drug sensitivity. Expression of the $I L-6$ gene was significantly decreased $24 \mathrm{~h}$ after $I L-6$ siRNA transfection in HAC-2 and JHOC-5 (Fig. 2B). The biological effect on cell proliferation was analyzed by cell count after transfection of $I L-6$ siRNA in CCC cell lines. No significant effect on cell proliferation was observed when compared to transfection of negative control siRNA (data not shown).

Although there was no change in proliferation, IL-6 inhibition did modulate downstream signaling. Stat3 is a major signaling effector of IL-6 and has been shown to be required for survival in multiple cancer models (15). Suppression of IL-6 reduced activation of Stat3 (pStat3) without affecting overall levels of Stat 3 or other components of the signaling pathway (IL-6R $\alpha$; Fig. 3A). Given these effects on a pro-survival pathway we next evaluated the susceptibility of CCC cell lines to cytotoxic agents after suppression of $I L-6 . I L-6$ siRNA transfected HAC-2 exhibited a statistically significant increase in cell death to both paclitaxal $\left(\mathrm{IC}_{50}\right.$ was $\left.4.78 \pm 0.21 \mu \mathrm{M}\right)$ and cisplatin $\left(\mathrm{IC}_{50}\right.$ was $\left.1.14 \pm 0.02 \mu \mathrm{M}\right)$ as compared with negative control siRNA transfected cells $\left(\mathrm{IC}_{50}\right.$ for paclitaxal was $9.82 \pm 0.41 \mu \mathrm{M}$ and for cisplatin $2.12 \pm 0.01 \mu \mathrm{M}$, respectively) (Fig. 3B). Similar results were obtained from JHOC-5 cells. These results indicate that inhibition of endogenous IL-6 in CCC cells decreases Stat 3 activation and sensitizes these cells to cytotoxic stress.

\section{Discussion}

Cytokine expression within a tumor microenvironment plays a fundamental role in cancer development and progression;
Table II. Clinicopathological characteristics of the 50 ovarian cancer samples by unsupervised hierarchical cluster group.

\begin{tabular}{lccc}
\hline Parameters & $\begin{array}{c}\text { Cluster A } \\
(\mathrm{n}=28)\end{array}$ & $\begin{array}{c}\text { Cluster B } \\
(\mathrm{n}=22)\end{array}$ & p-value \\
\hline Patient age & & & \\
$\quad$ 60 years & 23 & 18 & 0.733 \\
$>60$ years & 5 & 4 & \\
Histological type & & & \\
SA+EA & 24 & 5 & $<0.001$ \\
CCC & 4 & 17 & \\
FIGO stage & & 13 & 0.105 \\
I+II & 9 & 9 & \\
III+IV & 19 & & \\
Residual tumor & & 16 & \\
$\leq 1 \mathrm{~cm}$ & 17 & 6 & \\
$>1 \mathrm{~cm}$ & 11 & & \\
\hline
\end{tabular}

SA, serous adenocarcinoma; EA, endometrioid adenocarcinoma; CCC, clear cell carcinoma.

Table III. Cytokine genes differentially expressed among CCC vs. non-CCC.

\begin{tabular}{lccc}
\hline Gene & p-value & FDR & Permutation p-value \\
\hline$I L-6$ & $<1 \mathrm{e}-07$ & $<1 \mathrm{e}-07$ & $<1 \mathrm{e}-07$ \\
$I L-12 p 35$ & 0.0255652 & 0.105 & 0.0237 \\
$I L-1 \beta$ & 0.0262116 & 0.105 & 0.0249 \\
$I L-10$ & 0.0384981 & 0.115 & 0.0375
\end{tabular}

The four genes are significant at 0.05 level of the univariate test. Permutation p-values for significant genes were computed based on 10000 random permutations.

tumor cells that produce immunosuppressive cytokines can escape the host immune response. In ovarian cancer, several cytokines associated with cellular immunity were correlated to cancer development and patient prognosis, including TNF- $\alpha$, IFN- $\gamma$, IL-6, and MHC molecules (9). We report here that the expression profile of 12 pro- and anti-inflammatory cytokine genes in tumor could reliably distinguish the CCC histopathological classification of ovarian carcinoma from other epithelial subtypes. Overall evidence suggested CCC to have a Th-2 cytokine dominant expression pattern, including high levels of IL-6. In our series, 17 of 19 stage III/IV cases $(89 \%)$ in cluster A were SA and 12 of 13 stage I/II cases (92\%) in cluster B were CCC. It should also be noted that since $>50 \%$ of $\mathrm{CCC}$ is diagnosed at an early stage, as seen in this study $(67 \%)$, the marginal association between the expression signature and FIGO stage is likely the result of confounded representation of $\mathrm{CCC}$ in cluster $\mathrm{B}$.

CCC differs considerably from the other histological types of ovarian cancer with respect to its clinical and molecular characteristics $(5,16,19,20)$. With respect to cytokine expression signature and pathway activation, Yamaguchi et al 


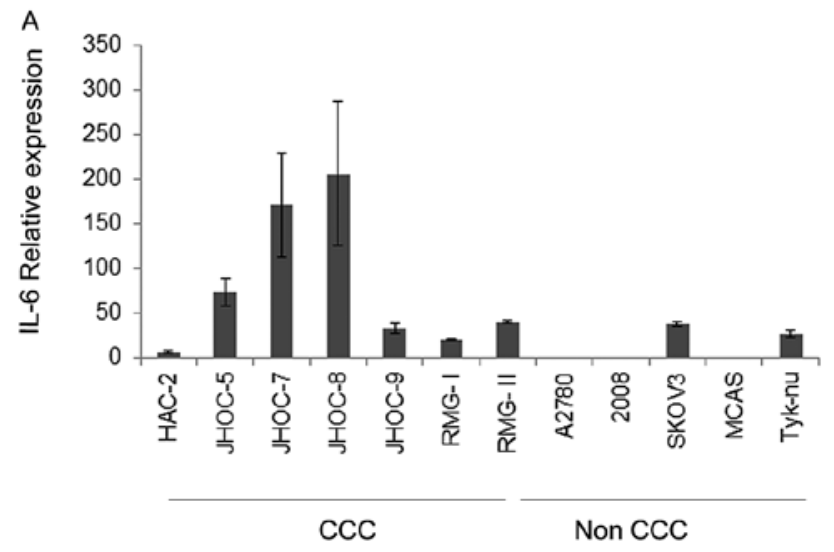

B

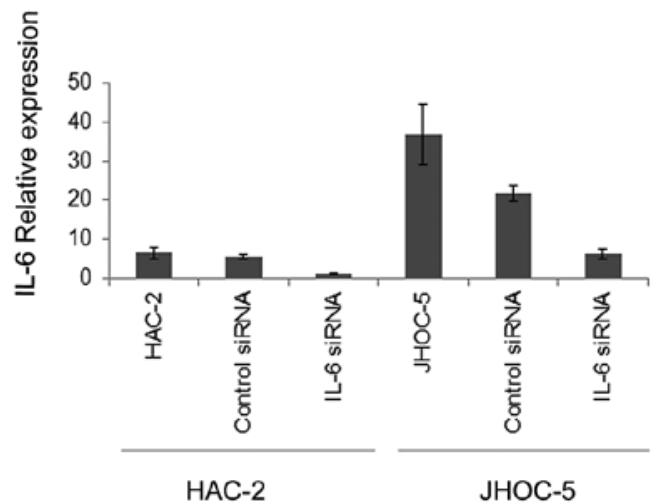

Figure 2. $I L-6$ expression and $I L-6$ siRNA transfection in ovarian cancer cell lines. Gene expression was quantified using the comparative method and normal ovarian tissue was used as a reference for each sample. Human 18S rRNA was used as an endogenous control. Data are shown as the mean \pm SD. (A) Relative expression of the $I L-6$ gene in seven CCC cell lines and five non-CCC cell lines. (B) $I L-6$ and negative control siRNA transfection in CCC cell lines. Expression of the $I L-6$ gene was decreased $24 \mathrm{~h}$ after $I L-6$ siRNA transfection in HAC-2 and JHOC- 5 cells.

A

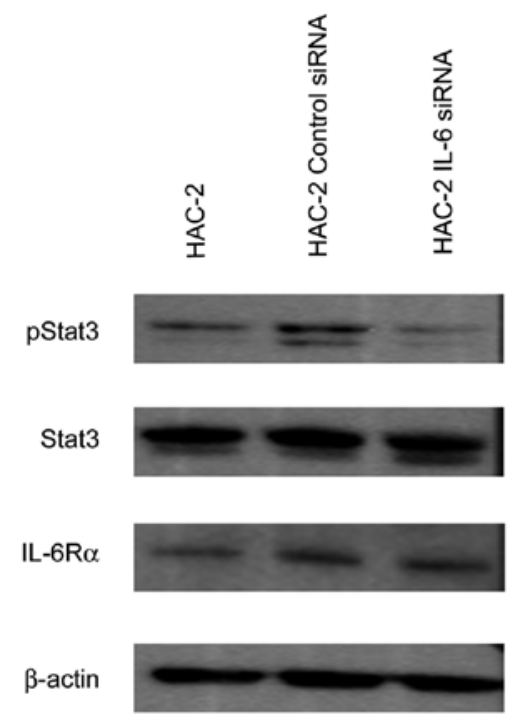

B
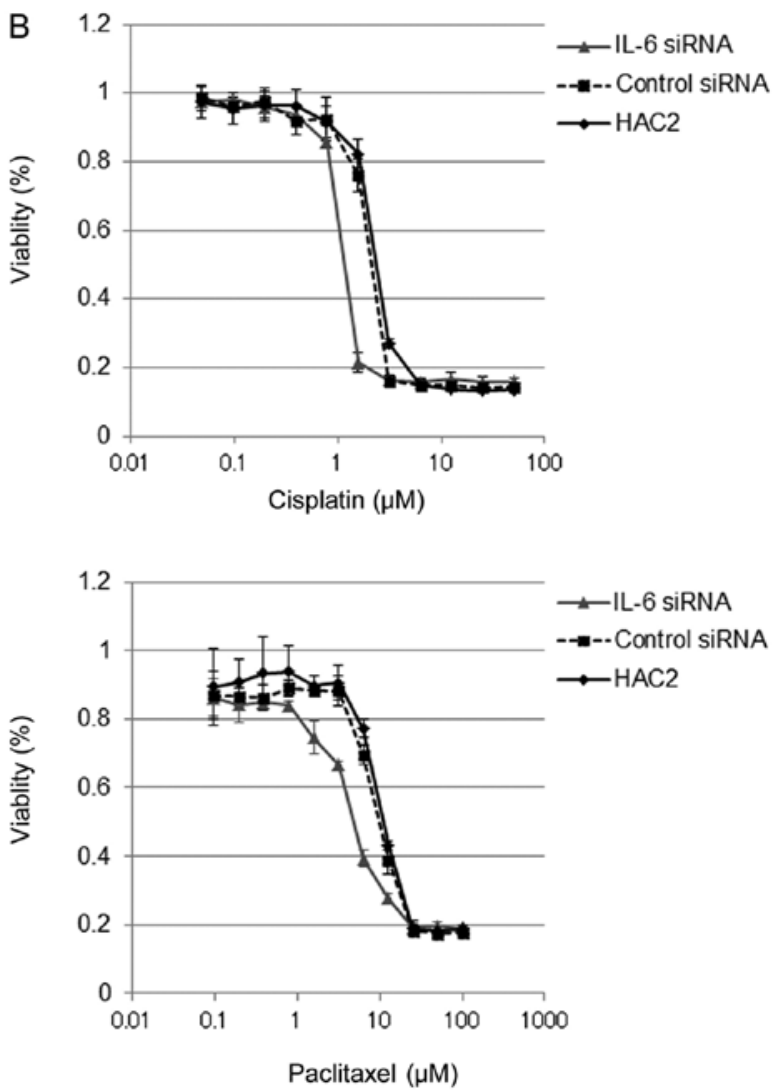

Figure 3. Effect of IL-6 expression on the responsiveness of CCC cell to paclitaxel and cisplatin. (A) Sensitivity to paclitaxel and cisplatin was measured by MTS assay in HAC-2. (B) Phosphorylated Stat3, total Stat3 and IL-6R $\alpha$ expression levels were analyzed in HAC-2 cells transfected with either $I L-6$ siRNA or negative control siRNA. $\beta$-actin was also used as loading control.

identified a CCC cell line specific gene signature from a large set of microarray data that contained $I L-6$, consistent with data presented here, as well as several previously known CCC markers such as $H N F-1 \beta$ (20). Our data further supports findings that implicate the activated Stat 3 signaling pathway in CCC cells $(19,20)$, with specific overexpression IL-6 in CCC compared to SA amongst the 12-cytokine panel examined and suppression of Stat 3 signaling when IL-6 was knocked down with siRNA. These findings also suggest that the tumor cells, rather than the host-microenvironment, is the source of IL-6 expression. This is in contrast to previous work with highgrade SA where a subset of this histological type is associated with tumor infiltrating lymphocytes (TILs) $(5,17,18)$. In these SA-specific studies the dominant cytokine expression pattern is presumed to be driven by the non-tumor microenvironment. However, given the modest number of samples in this study 
and the overall prevalence of TILs amongst high-grade SA, it seems unlikely that TILs alone are influencing the expression pattern of cytokines in the serous tumors examined here. Markedly, expression profiling has suggested that high-grade SA with the highest levels of IL-6 are not those with TILs (18).

One of the most clinically important biological features of CCC is its inherent chemoresistance, which is associated with its poor prognosis particularly at advanced stages. Several mechanisms involved in drug resistance in CCC have been proposed, however, the precise mechanisms underlying chemoresistance remain to be elucidated (16). In this study, siRNA-mediated IL-6 expression did not directly affect proliferation, however, we found that IL-6 inhibition did reduce Stat 3 activation and increase the sensitivity of CCC cell lines to both cisplatin and paclitaxel. IL-6 is a pleiotropic cytokine that plays important roles in the immune response and inflammation. Aberrant expression of $I L-6$ has been implicated in many types of cancer, and the role of IL- 6 in chemoresistance has been addressed in several malignancies, including multiple myeloma, renal cell carcinoma, cholangiocarcinoma, prostate cancer, and breast cancer (21). A clear link between an IL-6 rich tumor microenvironment and tumor progression or survival has been observed in other tumor models (15). Recent evidence indicates that autocrine and paracrine effects by constitutive production of IL-6 in ovarian cancer could be involved in the tumorigenic potential through the regulation of angiogenesis (22) or MMP secretion (23). These results suggest that a drug resistant phenotype in CCC may, in part, be explained by the activated autocrine IL-6 signaling pathway including Stat 3 activation. It is interesting to speculate on a biological link between recently discovered endometriosis associated ovarian cancer ARIDIA mutations $(24,25)$, chemoresistance, and the cytokine profile specific to CCC. Though not clearly established in this context, modulation of gene expression controlled by the SWI/SNF chromatin binding complex, specifically the BRG1 component, has been shown to be required for induction of cytokine genes, including IL-6, during inflammatory responses (26).

Finally, although SA and CCC behave like distinct entities, a chemoresistant mechanism converging around the IL-6 pathway may be highly relevant to both CCC and at least some high-grade SA. Wang et al showed that IL-6 secreted by serous type ovarian cancer cells might contribute to the chemoresistance through the downregulation of caspase-3 and increased expression of both multidrug resistance-related genes and apoptosis inhibitory proteins (27). High IL-6 levels in the serum and ascites of ovarian cancer have been found to be associated with poor prognosis and chemoresistance (21). Most studies on this topic, however, have not differentiated between histological subtypes. More recently, blockage of IL-6 signaling by a monoclonal anti-IL-6 antibody siltuximab (CNTO 328) with cytotoxic agent has been shown to disrupt cancer progression in serous ovarian cancer cell lines both in vitro and in mouse xenograft models (28). Siltuximab decreased Stat 3 phosphorylation and protein levels of downstream effectors including MCL-1, Bcl-XL, and Survivin in paclitaxel resistant ovarian cancer cell lines. This is consistent with decreased levels of phospho-Stat 3 and chemo-sensitization observed in this report. In the phase II clinical trial with platinum-resistant ovarian cancer, siltuximab had some therapeutic activity (29). Exposure of ovarian cancer cells to siltuximab had no effect on cell growth, also consistent with our siRNA-mediate $I L-6$ knockdown experiments. This has led to the hypothesis that the growth inhibitory effects of IL-6 knockdown may be evident only with tumor-stromal influences. Functional in vivo model systems paired with immunohistochemical and molecular analysis are needed to further refine the roles of various immune cells contributing to the molecular pathogenesis of ovarian cancer. In particular, the IL-6 related mechanism of chemo-resistance in both CCC and high-grade SA, direct inhibition of IL-6, IL-6R or other downstream signaling effectors, such as Stat3, should not be overlooked. Nonetheless, the results obtained in this study support the idea of targeting the IL-6 signaling pathway in a combination therapy approach sensitizing CCC tumor cells in particular to current gold-standard chemotherapies already in use for ovarian carcinoma.

\section{Acknowledgements}

This work was supported in part by Grants-in-Aid for Scientific Research from Japan Society for the Promotion of Science to N.Y., A.O., K.Y., and S.T., and by the Japanese Ministry of Health, Labour and Welfare to K.O. Analyses were performed using BRB ArrayTools developed by Dr Richard Simon and Amy Peng Lam.

\section{References}

1. Vaughan S, Coward JI, Bast RC Jr, et al: Rethinking ovarian cancer: recommendations for improving outcomes. Nat Rev Cancer 23: 719-725, 2011.

2. Köbel M,Kalloger SE, Boyd N, et al: Ovarian carcinoma subtypes are different diseases: implications for biomarker studies. PLoS Med 5: e232, 2008.

3. Swenerton KD, Santos JL, Gilks CB, et al: Histotype predicts the curative potential of radiotherapy: the example of ovarian cancers. Ann Oncol 22: 341-347, 2010.

4. Gilks CB, Ionescu DN, Kalloger SE, et al: Tumor cell type can be reproducibly diagnosed and is of independent prognostic significance in patients with maximally debulked ovarian carcinoma. Hum Pathol 39: 1239-1251, 2008.

5. Anglesio MS, Carey MS, Köbel M, et al: Clear cell carcinoma of the ovary: A report from the first Ovarian Clear Cell Symposium, June 24th, 2010 (Review). Gynecol Oncol 121: 407-415, 2010.

6. Takakura S, Takano M, Takahashi F, et al: Japanese Gynecologic Oncology Group. Randomized phase II trial of paclitaxel plus carboplatin therapy versus irinotecan plus cisplatin therapy as first-line chemotherapy for clear cell adenocarcinoma of the ovary: a JGOG study. Int J Gynecol Cancer 20: 240-247, 2010.

7. Shimada M, Kigawa J, Ohishi Y, et al: Clinicopathological characteristics of mucinous adenocarcinoma of the ovary. Gynecol Oncol 113: 331-334, 2009.

8. Schreiber RD, Old LJ and Smyth MJ: Cancer immunoediting: integrating immunity's roles in cancer suppression and promotion (Review). Science 331: 1565-1570, 2011.

9. Nelson BH: The impact of T-cell immunity on ovarian cancer outcomes (Review). Immunol Rev 222: 101-116, 2008.

10. Kusuda T, Shigemasa K, Arihiro K, et al: Relative expression levels of Th1 and Th2 cytokine mRNA are independent prognostic factors in patients with ovarian cancer. Oncol Rep 13: 1153-1158, 2005.

11. Marth C, Fiegl H,Zeimet AG, et al: Interferon-gamma expression is an independent prognostic factor in ovarian cancer. Am J Obstet Gynecol 191: 1598-1605, 2004.

12. Seike M, Yanaihara N, Bowman ED, et al: Use of a cytokine gene expression signature in lung adenocarcinoma and the surrounding tissue as a prognostic classifier. J Natl Cancer Inst 99: 1257-1269, 2007. 
13. Kurman RJ and Shih IeM: Molecular pathogenesis and extraovarian origin of epithelial ovarian cancer - shifting the paradigm. Hum Pathol 42: 918-931, 2001.

14. Bustin SA: Absolute quantification of mRNA using real-time reverse transcription polymerase chain reaction assays. J Mol Endocrinol 25: 169-193, 2000.

15. Li N, Grivennikov SI and Karin M: The unholy trinity: inflammation, cytokines, and STAT3 shape the cancer microenvironment. Cancer Cell 12: 429-431, 2011.

16. Itamochi H, Kigawa J and Terakawa N: Mechanisms of chemoresistance and poor prognosis in ovarian clear cell carcinoma (Review). Cancer Sci 99: 653-658, 2008.

17. Tsiatas ML, Gyftaki R, Liacos C, et al: Study of T lymphocytes infiltrating peritoneal metastases in advanced ovarian cancer: associations with vascular endothelial growth factor levels and prognosis in patients receiving platinum-based chemotherapy. Int J Gynecol Cancer 19: 1329-1334, 2009.

18. Tothill RW, Tinker AV, George J, et al: Novel molecular subtypes of serous and endometrioid ovarian cancer linked to clinical outcome. Clin Cancer Res 14: 5198-5208, 2008.

19. Anglesio MS, George J, Kulbe H, et al: IL6-STAT3-HIF signaling and therapeutic response to the angiogenesis inhibitor sunitinib in ovarian clear cell cancer. Clin Cancer Res 17: 2538-2548, 2011.

20. Yamaguchi K, Mandai M, Oura T, et al: Identification of an ovarian clear cell carcinoma gene signature that reflects inherent disease biology and the carcinogenic processes. Oncogene 29: 1741-1752, 2010.

21. Hong DS, Angelo LS and Kurzrock R: Interleukin-6 and its receptor in cancer: implications for translational therapeutics (Review). Cancer 110: 1911-1928, 2007.
22. Nilsson MB, Langley RR and Fidler IJ: Interleukin-6, secreted by human ovarian carcinoma cells, is a potent proangiogenic cytokine. Cancer Res 65: 10794-10800, 2005.

23. Rabinovich A, Medina L, Piura B, Segal S and Huleihel M: Regulation of ovarian carcinoma SKOV-3 cell proliferation and secretion of MMPs by autocrine IL-6. Anticancer Res 27: 267-272, 2007.

24. Jones S, Wang TL, Shih IeM, et al: Frequent mutations of chromatin remodeling gene ARID1A in ovarian clear cell carcinoma. Science 330: 228-231, 2010.

25. Wiegand KC, Shah SP, Al-Agha OM, et al: ARID1A mutations in endometriosis-associated ovarian carcinomas. N Engl J Med 363: 1532-1543, 2010

26. Cullen SJ, Ponnappan S and Ponnappan U: Catalytic activity of the proteasome fine-tunes Brg1-mediated chromatin remodeling to regulate the expression of inflammatory genes. Mol Immunol 47: 600-605, 2009.

27. Wang Y, Niu XL, Qu Y, et al: Autocrine production of interleukin-6 confers cisplatin and paclitaxel resistance in ovarian cancer cells. Cancer Lett 295: 110-123, 2010.

28. Guo Y, Nemeth J, O'Brien C, et al: Effects of siltuximab on the IL-6-induced signaling pathway in ovarian cancer. Clin Cancer Res 16: 5759-5769, 2010.

29. Coward J, Kulbe H, Chakravarty P, et al: Interleukin-6 as a therapeutic target in human ovarian cancer. Clin Cancer Res 17: 6083-6096, 2011. 